\title{
MELOLONTHIDAE NOCTURNOS (COLEOPTERA) RECOLECTADOS EN LA ZONA AGRÍCOLA AGAVERA DE JALISCO, MÉXICO
}

\author{
Gabriel Antonio Lugo-García, ${ }^{* 1}$ Laura Delia Ortega-Arenas, ${ }^{1}$ Héctor \\ GonZález-Hernández, ${ }^{1}$ Agustín Aragón-García, ${ }^{2}$ Jesús Romero-NÁpoles, ${ }^{1}$ \\ Ramón Rubio-CoRTÉs ${ }^{3}$ y Miguel Ángel Morós ${ }^{4}$ \\ ${ }^{1}$ Entomología, Campus Montecillo. Colegio de Postgraduados, 56230. Montecillo, Estado de México. \\ *<antoniolugo@colpos.mx> \\ ${ }^{2}$ Agroecología y Ambiente, Instituto de Ciencias, Benemérita Universidad Autónoma de Puebla. \\ 72570. San Manuel Puebla, Puebla. \\ ${ }^{3}$ Tequila Sauza S.A. de C. V., Depto. de Investigación y Desarrollo, Rancho El Indio. 46400. Tequila, \\ Jalisco, México. \\ ${ }^{4}$ Red Biodiversidad y Sistemática, Instituto de Ecología A. C. Apartado Postal 63, Xalapa, Veracruz, \\ 91000.<miguel.moron@inecol.edu.mx>
}

Lugo-García, G. A., L. D. Ortega-Arenas, H. González-Hernández, A. Aragón-García, J. Romero-Nápoles, R. Rubio-Cortés \& M. Á. Morón. 2011. Melolonthidae nocturnos (Coleoptera) recolectados en la zona agrícola agavera de Jalisco, México. Acta Zoológica Mexicana (n. s.), 27(2): 341-357.

RESUMEN. Con la finalidad de conocer la fauna de melolóntidos nocturnos en las zonas agaveras de mayor importancia en el estado de Jalisco, se realizaron capturas mensuales de mayo a agosto de 2007, utilizando una trampa de luz fluorescente negra tipo embudo, en Ixtlahuacán del Río, Tepatitlán de Morelos y San Juanito de Escobedo, Jalisco, México. Se recolectaron un total de 57,263 especímenes pertenecientes a 20 especies. Phyllophaga ravida (Blanchard) (58.7\%), Paranomala cincta (Say) (14.1\%), Phyllophaga misteca (Bates) (11.6), P. dentex (Bates) (2.5\%) y Paranomala hoepfneri (Bates) (1.8\%) fueron las especies mejor representadas. La mayor abundancia se presentó en el mes de junio (96\%) y la menor en el mes de julio (0.02\%). La mayor riqueza específica (17 especies) se presentó en Tepatitlán de Morelos. Se incluye una clave para identificar las especies encontradas.

Palabras clave: Phyllophaga, Cyclocephala, Paranomala, Agave tequilero, gallina ciega.

Lugo-García, G. A., L. D. Ortega-Arenas, H. González-Hernández, A. Aragón-García, J. RomeroNápoles, R. Rubio-Cortés \& M. Á. Morón. 2011. Nocturnal Melolonthidae (Coleoptera) collected in agave fields of Jalisco, Mexico. Acta Zoológica Mexicana (n. s.), 27(2): 341-357.

ABSTRACT. In order to know the nocturnal fauna of Melolonthidae from the main agave tequilero fields in Jalisco, Mexico, monthly captures of adults were carried out from May to August 2007, using a

Recibido: 10/05/2010; aceptado: 24/11/2010. 
fluorescent light black trap, funnel type, in Ixtlahuacán del Río, Tepatitlán de Morelos and San Juanito de Escobedo, Jalisco, Mexico. A total of 57,263 specimens included in 20 species were collected. Phyllophaga ravida (Blanchard) (58.7\%), Paranomala cincta (Say) (14.1\%), Phyllophaga misteca (Bates) (11.6), P. dentex (Bates) (2.5\%) and Paranomala hoepfneri (Bates) (1.8\%) were the best represented species. The highest abundance was registered in June (96\%) and the lowest capture in July. The greatest species richness (17 species) was recorded in Tepatitlán de Morelos. A key to identify the recorded species is included.

Key words: Phyllophaga, Cyclocephala, Paranomala, Tequila agave, White grubs.

\section{INTRODUCCIÓN}

La familia Melolonthidae está representada en México por seis subfamilias, 23 tribus, 19 subtribus, 119 géneros y 1,040 especies (Blackwelder \& Arnett 1974, Endrödi 1985, Morón 2004). Con los datos disponibles hasta el momento se puede señalar que los estados con mayor diversidad de Melolonthidae son Chiapas, Oaxaca y Veracruz, en donde se han registrado 368, 223 y 220 especies, respectivamente (Morón et al. 1997; Pacheco et al. 2008). Para el estado de Jalisco se han registrado 310 especies (Navarrete-Heredia et al. 2001), donde Phyllophaga es el género mejor representado con 50 especies (Morón \& Rivera 2005); este género es de los más conocidos debido a la gran diversidad de cultivos que afecta (Aragón \& Morón 1998).

En México los adultos de "gallina ciega" se conocen comúnmente como "escarabajos de mayo", "mayates de junio" o "escarabajos sanjuaneros", "xkumuk", "ronrones", “jobotos" (Aragón et al. 2006, Ramírez et al. 2004, Morón et al. 1997); generalmente estos escarabajos se alimentan con el follaje de árboles y arbustos silvestres, como encinos (Quercus spp.) y “chilca” (Senecio sp.) (Morón et al. 1997, Ramírez-Salinas \& Castro-Ramírez 2000, 2006). Se han realizado algunos estudios faunísticos de las especies de Melolonthidae en regiones agrícolas de Nayarit, Puebla, Chiapas y Michoacán (Aragón et al. 1998, 2001, Morón et al. 1998, Nájera 1998, Castro-Ramírez et al. 2005). En Jalisco, Nájera (1993) estudió en la región centro del estado a los coleópteros asociados al cultivo de maíz y Magaña \& Rivera (1998) documentaron la abundancia de los melolóntidos nocturnos de un bosque de pinoencino del municipio de Atenguillo; mientras que López \& Rivera (1998) realizaron el registro de melolóntidos en un bosque mesófilo de montaña en la Sierra de Manantlán.

Aun cuando el agave tequilero Agave tequilana Weber var. azul (Agavaceae) es uno de los cultivos más importantes en el estado de Jalisco (Luna 1991) pocos son los estudios que se han realizado para conocer la diversidad de melolóntidos asociados al cultivo, y su potencial como plagas subterráneas. Entre ellos, Angelina (2004) al realizar un estudio en los Altos de Jalisco encontró que Cyclocephala comata Bates, 1888, Phyllophaga polyphylla (Bates, 1888) y Phyllophaga misteca (Bates,1888) fueron las especies más abundantes. Para ampliar dicha información, en el presente 
estudio se realizó una evaluación de la fauna de melolóntidos que habita en otras regiones donde se cultiva el agave tequilero en Jalisco, que contribuye al inventario faunístico del estado e incluye una clave dicotómica para facilitar la identificación de las especies registradas.

\section{MATERIALES Y MÉTODOS}

Esta investigación se realizó en las localidades de Ixtlahuacán del Río $\left(20^{\circ} 48^{\prime} \mathrm{N}\right.$, $103^{\circ} 11^{\prime} \mathrm{O}$ y $\left.1642 \mathrm{~m}\right)$, Tepatitlán de Morelos $\left(20^{\circ} 44^{\prime} \mathrm{N}, 102^{\circ} 44 \mathrm{~W}\right.$ y $1820 \mathrm{~m}$ ) y San Juanito de Escobedo ( $20^{\circ} 43^{\prime} \mathrm{N}, 104^{\circ} 04^{\prime} \mathrm{W}$ y $\left.1360 \mathrm{~m}\right)$, Jalisco, México, porque en dichos sitios los productores y los técnicos de las empresas tequileras otorgaron facilidades para realizar los trabajos de campo.

Durante los meses de mayo a agosto de 2007 se realizaron recolectas nocturnas de melolóntidos utilizando una trampa de luz fluorescente negra de 20 watts tipo embudo, conectada con un recipiente colector de 19 litros de capacidad y abastecida con una batería para automóvil (Aragón et al. 2008). Se utilizó una trampa por localidad, la cual se colocó en el centro de la parcela de cultivo en las primeras horas de la noche (19:00 h) y fue retirada cuatro horas después (23:00 h). La captura se realizó una noche por mes. En forma complementaria, y debido a que algunas especies no son atraídas por la luz, se realizaron prospecciones sobre plantas hospederas a las orillas del cultivo con ayuda de linternas portátiles para localizar adultos alimentándose o apareándose (Méndez et al. 2005). Los insectos capturados se conservaron en alcohol etílico al $70 \%$, se separaron y contaron antes de montar en alfileres entomológicos series representativas de cada especie. La determinación del material se realizó con ayuda de las claves dicotómicas para la fauna de Coleoptera Scarabaeoidea de los estados de Hidalgo, Morelos y Nayarit (Morón 1994, Deloya \& Morón 1994, Morón et al. 1996). El material estudiado se depositó en las colecciones entomológicas del Colegio de Postgraduados, Montecillo, Edo de México; en la Empresa Tequila Sauza, Tequila, Jalisco y del Departamento de Investigación en Ciencias Agrícolas, Instituto de Ciencias, Benemérita Universidad Autónoma de Puebla, en la Ciudad de Puebla. Las determinaciones fueron corroboradas por el Dr. Miguel Ángel Morón, del Instituto de Ecología A.C. en Xalapa, Veracruz.

\section{RESULTADOS}

En las trampas de luz instaladas en las parcelas de agave tequilero en las tres localidades citadas se capturaron un total de 57,263 ejemplares que representan a tres subfamilias, nueve tribus, 10 géneros y 20 especies de la familia Melolonthidae (Cuadro 1). Las especies mejor representadas fueron Phyllophaga ravida (58.7\%), Paranomala cincta (14.1\%), Phyllophaga misteca (11.6), Diplotaxis trapezifera (6\%), Phyllophaga dentex (2.5\%) y Paranomala hoepfneri (1.8\%). La mayor abundancia se presentó 
Cuadro 1. Especies de Melolonthidae recolectadas con trampa de luz fluorescente negra en cultivo de agave tequilero en tres localidades del estado de Jalisco, México.

\begin{tabular}{|c|c|c|c|}
\hline Subfamilias & Tribus & Especies & Núm. Adultos \\
\hline \multirow[t]{8}{*}{ Melolonthinae } & Melolonthini & Phyllophaga ravida & 33,663 \\
\hline & & Ph. polyphylla & 1,208 \\
\hline & & Ph.misteca & 6,659 \\
\hline & & Ph.dentex & 1,427 \\
\hline & & Ph. aff.nogueirai & 2 \\
\hline & & Ph.aff.gigantissima & 2 \\
\hline & Diplotaxini & Diplotaxis trapezifera & 3,440 \\
\hline & Macrodactylini & Isonychus arizonensis & 36 \\
\hline \multirow[t]{7}{*}{ Dynastinae } & Cyclocephalini & Cyclocephala comata & 743 \\
\hline & & C.lunulata & 31 \\
\hline & & C.sinaloae & 3 \\
\hline & Pentodontini & Ligyrus sallei & 441 \\
\hline & Oryctini & Strategus aloeus & 79 \\
\hline & & Xyloryctes corniger & 12 \\
\hline & Dynastini & Golofa pusilla & 37 \\
\hline \multirow[t]{5}{*}{ Rutelinae } & Anomalini & Paranomala hoepfneri & 1,295 \\
\hline & & Pa.cincta & 8,054 \\
\hline & & Pa.micans & 4 \\
\hline & & Pa.inconstans & 126 \\
\hline & Rutelini & Pelidnota virescens & 1 \\
\hline total & & & 57,263 \\
\hline
\end{tabular}

en el mes de junio (96\%) lo cual coincidió con el inicio del periodo de lluvias en la región (Cuadro 2). A continuación se presenta la composición específica de cada género y se proporcionan datos básicos sobre su biología, fenología y distribución. En el Apéndice 1 se incluye una clave para identificar a las 20 especies.

\section{Melolonthinae, Melolonthini}

Phyllophaga Harris. Se registraron seis especies de los subgéneros Triodonyx y Phyllophaga (s.str) (sensu Morón 1986). El mayor número de especies se presentó en Tepatitlán, donde se obtuvieron seis especies (Cuadro 2) y el menor número de especies correspondió a Ixtlahuacán del Río y San Juanito de Escobedo, donde se registraron únicamente tres especies de este género en cada localidad. 
Phyllophaga (s.str..) ravida (Blanchard, 1850). Esta fue la especie más abundante en la región de Los Altos; de acuerdo con Díaz et al. (2006) y Nájera (1993) es la especie más ampliamente distribuida en dicha región y en general en el país. En este trabajo fue abundante en Tepatitlán, Jalisco, donde durante junio se recolectaron 31,006 ejemplares (Cuadro 2), de los cuales el 85\% fueron hembras, lo cual puede confirmar que los machos son poco atraídos por la luz, ya que éste resultado coincide con lo registrado en Cuautinchán, Puebla por Aragón et al. (2008) quienes observaron que en una muestra de 90,289 ejemplares capturados en trampa de luz fluorescente negra el $89 \%$ fueron hembras. Con frecuencia sus larvas se encuentran asociadas a los cultivos de maíz, caña de azúcar, pastos y hortalizas (Morón 2003). Se considera una de las plagas subterráneas más importantes en México (Morón et al. 1997, 1998). En el estado de Puebla los adultos se han recolectado directamente del follaje de "encino" (Quercus sp.) durante junio, julio y agosto (Aragón \& Morón 2000). Se distribuye en Aguascalientes, Chiapas, Chihuahua, Colima, Distrito Federal, Durango, Guerrero, Hidalgo, Jalisco, México, Michoacán, Morelos, Nayarit, Oaxaca, Puebla, Sinaloa y Veracruz (Morón 2003).

Phyllophaga (s.str.) polyphylla (Bates, 1888). Se recolectaron 1,190 ejemplares atraídos por la luz el 12 de junio de 2007 en Tepatitlán y sólo 18 ejemplares en San Juanito de Escobedo, el 20 de junio de 2007 (Cuadro 3). Nájera (1993) registró a los adultos de esta especie con hábitos diurnos en Ameca, Jalisco donde desarrollaron su máxima actividad de vuelo entre 7:30 y 10:00 hrs. Esta especie se distribuye en los estados de Aguascalientes, Jalisco y Michoacán (Morón 2003).

Phyllophaga (s.str.) dentex (Bates, 1888). Durante junio en Tepatitlán se recolectaron 1,327 ejemplares yen San Juanito de Escobedo solo 82 individuos.. Encabeza al complejo "dentex" del grupo "ravida" (Morón 1986) y tiene una amplia distribución en Chihuahua, Durango, Guanajuato, Guerrero, Hidalgo, Jalisco, México, Michoacán, Nayarit, Puebla, Sinaloa, Sonora, y en Arizona E.U.A. (Morón 2003).

Phyllophaga (s.str.) misteca (Bates, 1888). En San Juanito de Escobedo se colectaron 3,202 ejemplares y 3,358 en Tepatitlán en junio. Esta especie forma parte del grupo "anodentata" junto con otras 20 especies. Sus larvas se alimentan con raíces de maíz, pastos (Morón 2003) y se encuentran en el suelo de los cultivos de agave tequilero en Los Altos de Jalisco, pero no se ha observado que se alimenten con raíces de agave (Angelina 2004). Se ha recolectado en los estados de Durango, Jalisco, México, Oaxaca, Puebla, San Luis Potosí, Tlaxcala y Zacatecas (Morón 2003).

Phyllophaga (Triodonyx) aff. nogueirai Warner \& Morón, 1992. Se recolectaron dos machos el 12 de junio de 2007, en Tepatitlán, Jalisco. Dicha especie sólo se conoce de Jalisco (Morón 2003).

Phyllophaga (T.) aff. gigantissima Saylor, 1942. Se recolectó un macho el 12 de junio de 2007, en Tepatitlán, Jalisco (Cuadro 2). Dicha especie se ha registrado en los estados de Nayarit y Sinaloa,(Morón 2003). 
Cuadro 2. Registro de la abundancia mensual de las especies de Melolonthidae en Tepatitlán, Jalisco, México. (Todos los registros en trampa de luz negra, excepto C. comata por colecta directa).

\begin{tabular}{lrrr}
\hline Especies & Mayo & Junio & Agosto \\
\hline Phyllophaga dentex & 0 & 1,327 & 0 \\
Ph. polyphylla & 0 & 1,190 & 0 \\
Ph. ravida & 0 & 31,006 & 61 \\
Ph. misteca & 10 & 3,358 & 0 \\
Ph. (Triodonyx) aff. nogueirai & 0 & 2 & 0 \\
Ph. (Triodonyx) aff. gigantissima & 0 & 1 & 0 \\
Diplotaxis trapezifera & 77 & 605 & 5 \\
Cyclocephala comata & 3 & 588 & 0 \\
C. sinaloae & 0 & 3 & 0 \\
Paranomala cincta & 0 & 8,040 & 1 \\
Paranomala hoepfneri & 0 & 479 & 0 \\
Paranomala inconstans & 0 & 52 & 0 \\
Paranomala micans & 0 & 2 & 0 \\
Golofa pusilla & 0 & 25 & 0 \\
Strategus aloeus & 0 & 40 & 0 \\
Xyloryctes corniger & 0 & 2 & 0 \\
Ligyrus sallei & 0 & 0 & 5 \\
Total & 90 & 46,720 & 72 \\
\hline
\end{tabular}

\section{Melolonthinae, Diplotaxini}

Diplotaxis trapezifera Bates, 1887. Esta especie presentó su mayor actividad de vuelo en mayo y junio; su abundancia fue variable en las regiones de estudio (Cuadro 3). Distribución: Chiapas, Colima, Durango, Guerrero, Jalisco, Morelos, Nayarit, Oaxaca, Sinaloa, San Luis Potosí, Veracruz y Yucatán (Morón et al. 1997).

\section{Melolonthinae: Macrodactylini}

Isonychus arizonensis Howden, 1959. En San Juanito de Escobedo, atraídos por las trampas de luz en junio se obtuvieron 34 ejemplares de esta especie; y en Ixtlahuacán del Río únicamente se colectó una pareja. Esta es una especie con amplia distribución en las vertientes internas y externas de las montañas occidentales de México, en Jalisco se ha citado para las áreas de Autlán y Manantlán (Morón et al. 1997).

\section{Rutelinae, Rutelini}

Pelidnota virescens Burmeister, 1844. De esta especie solamente se recolectó un macho el 20 de junio de 2007 (Cuadro 3). Según Morón et al. (1997) los adultos se 
alimentan del follaje de Guazuma ulmifolia (Sterculiaceae) y sus larvas se han encontrado en las raíces podridas de árboles de mango y troncos derribados de Anona. Es una especie con amplia distribución en la vertiente del Pacífico Mexicano, desde el sur de Sonora hasta Tehuantepec, que penetra por la cuenca del Balsas.

\section{Rutelinae, Anomalini}

Paranomala hoepfneri (Bates, 1888). En junio se obtuvieron 1,294 individuos en las localidades de estudio. En México se encuentra distribuida entre los 800 y $1200 \mathrm{~m}$ de altitud en Chiapas, México, Morelos, Oaxaca y Puebla (Morón et al. 1997, Aragón et al. 2001). Nuevo registro de distribución para Jalisco.

Paranomala cincta (Say, 1835). Esta especie se colectó en gran abundancia en Tepatitlán donde el registro más numeroso se presentó en junio con 8,040 ejemplares (Cuadro 2). Las larvas se desarrollan en el suelo consumiendo humus o raíces de diversas plantas como maíz, caña de azúcar y frijol. Se distribuye en casi todo el país, exceptuando la Península de Baja California y la Meseta del Norte (Morón et al. 1997).

Paranomala micans (Burmeister, 1847). Se obtuvieron únicamente cuatro ejemplares de esta especie en junio, dos en Ixtlahuacán del Río y dos en Tepatitlán. Morón

Cuadro 3. Registro de la abundancia mensual de las especies de Melolonthidae en San Juanito de Escobedo, Jalisco, México. (Todos los registros en trampa de luz negra, excepto C. comata por colecta directa).

\begin{tabular}{lrrrr}
\hline Especies & Mayo & Junio & Julio & Agosto \\
\hline Phyllophaga dentex & 0 & 82 & 0 & 0 \\
Ph. polyphylla & 0 & 18 & 0 & 0 \\
Ph. ravida & 0 & 2558 & 10 & 0 \\
Ph. misteca & 0 & 3202 & 0 & 0 \\
Diplotaxis trapezifera & 27 & 1123 & 0 & 0 \\
Cyclocephala comata & 0 & 18 & 0 & 0 \\
Paranomala hoepfneri & 0 & 791 & 0 & 0 \\
Paranomala inconstans & 0 & 74 & 0 & 0 \\
Golofa pusilla & 0 & 12 & 0 & 0 \\
Strategus aloeus & 0 & 34 & 5 & 0 \\
Xyloryctes corniger & 0 & 10 & 0 & 0 \\
Isonychus arizonensis & 0 & 34 & 0 & 0 \\
Pelidnota virescens & 0 & 1 & 0 & 0 \\
Total & 27 & 7957 & 15 & 0 \\
\hline
\end{tabular}


et al. (1997) indican que esta especie se ha registrado en Amatitán, Jalisco; sus larvas se alimentan de raíces y se han observado en cultivos de maíz y caña de azúcar.

Paranomala inconstans (Burmeister, 1847). Se recolectaron 126 adultos atraídos a la trampa de luz en junio en Tepatitlán, mientras que en Ixtlahuacán del Río y San Juanito se recolectaron 52 y 74 ejemplares respectivamente. Esta especie tiene amplia distribución en Nayarit, Guerrero, Guanajuato y Jalisco (Autlán y Manantlan) (Morón et al. 1997). Se desconocen los hábitos de alimentación de larvas y adultos.

\section{Dynastinae, Cyclocephalini}

Cyclocephala comata Bates, 1888. Los adultos de esta especie no fueron atraídas por las trampas de luz; se les recolectó durante junio y julio en Ixtlahuacán del Río cuando volaban hacia los árboles de fresno (Fraxinus $s p$.) en donde se alimentaban y copulaban (Cuadro 4). En Tepatitlán copulaban en árboles de huizache (Acacia sp. Fabaceae) y en plantas de agave tequilero pero no se les observo alimentándose. Morón y colaboradores (1997) indican que la especie se encuentra en Michoacán, Durango, México, Oaxaca y Jalisco; en este último se le registró asociada al cultivo de maíz en la región de Los Altos y en la región centro del estado (Nájera, 1993, Díaz et al. 2006).

Cyclocephala sinaloae Howden y Endrödi, 1966. Estuvo representada únicamente por tres ejemplares recolectados en Tepatitlán en junio. Esta especie forma parte de la fauna restringida entre los estados de Sinaloa y Jalisco (Morón et al. 1998).

Cyclocephala lunulata Burmeister, 1847. Se recolectaron 31 ejemplares en junio en Ixtlahuacán del Río atraídos por la trampa de luz (Cuadro 4). Según Díaz et al. (2006) las larvas de C. lunulata se han encontrado asociadas al cultivo de maíz en Los Altos de Jalisco, principalmente en suelos con un alto contenido de materia orgánica. Es una especie común con amplia distribución en México (excepto en Baja California y los estados del norte), Centro y Sudamérica (Morón et al. 1997).

\section{Dynastinae, Pentodontini}

Ligyrus sallei Bates, 1888. Esta especie se recolectó sólo en dos de los tres sitios muestreados (Ixtlahuacán del Río 436 especímenes; Tepatitlán 5 ejemplares) y las capturas se hicieron en agosto con la trampa de luz. Morón (1994) y Morón et al. (1998) refieren que esta especie es más común en las partes cercanas a las montañas o zonas forestadas. Aragón et al. (2001) indican que las larvas de esta especie se alimentan principalmente de materia orgánica y que es común encontrarlas en estiércol humificado y desechos agrícolas. Para el estado de Jalisco se encuentra reportada para Puerto Vallarta, Ameca, Autlán, Cocula, Guadalajara, Chapala y Volcán de Colima (Morón et al. 1997). 


\section{Dynastinae, Oryctini}

Xyloryctes corniger Bates, 1843. De esta especie se recolectaron pocos ejemplares (San Juanito de Escobedo 10 especímenes, Tepatitlán 2 ejemplares), las capturas se hicieron en junio con una trampa de luz. Se distribuye en Guerrero, Jalisco, México, Michoacán, Morelos, Nayarit y Oaxaca; en Jalisco se ha citado para Autlán y Zapopan (Morón et al. 1997).

Strategus aloeus (Linné, 1758). Se recolectaron ejemplares sólo en dos de las tres localidades (San Juanito de Escobedo 39 individuos, Tepatitlán 40 ejemplares). De acuerdo con Morón et al. (1998) es fácil recolectar a esta especie en junio, julio y agosto en Nayarit. Morón et al. (1997) indican que esta especie tiene amplia distribución en México (excepto Baja California) y que tanto los adultos como las larvas se alimentan de materia orgánica del suelo, raíces, tubérculos o tallos subterráneos y en ocasiones se les ha encontrado barrenando tejidos xilosos. Pérez-Domínguez (2006) la reporta como una plaga importante del agave debido a los daños que los adultos ocasionan en la base de las plantas.

\section{Dynastinae, Dynastini}

Golofa pusilla Arrow, 1911. Fue una especie poco abundante ya que en la localidad de San Juanito de Escobedo sólo se recolectaron 12 ejemplares y en Tepatitlán 25. Según Morón et al. (1998) las larvas se desarrollan en el suelo de los cañaverales de Tepic, Nayarit; sin embargo, no se ha comprobado que se alimenten de las raíces de

Cuadro 4. Registro de la abundancia mensual de las especies de Melolonthidae en Ixtlahuacán del Río, Jalisco, México. (Todos los registros en trampa de luz negra, excepto C. comata por colecta directa).

\begin{tabular}{lrrr}
\hline Especies & Mayo & Junio & Agosto \\
\hline Phyllophaga dentex & 0 & 18 & 0 \\
Ph. Ravida & 0 & 25 & 0 \\
Ph. misteca & 0 & 89 & 0 \\
Diplotaxis trapezifera & 1601 & 2 & 0 \\
Cyclocephala comata & 0 & 137 & 0 \\
C. lunulata & 0 & 31 & 0 \\
Paranomala cincta & 0 & 13 & 0 \\
Pa. hoepfneri & 0 & 25 & 0 \\
Pa. micans & 0 & 2 & 0 \\
Isonychus arizonensis & 0 & 2 & 0 \\
Ligyrus sallei & 0 & 0 & 436 \\
Total & 1601 & 344 & 436 \\
\hline
\end{tabular}


este cultivo. Es una especie con amplia distribución en los estados de Colima, Jalisco (Ameca, Ahualulco, Guadalajara y Zapopan), México, Michoacán, Morelos y Nayarit (Morón et al. 1997).

\section{DISCUSIÓN}

En cuanto a la fenología de los melolóntidos capturados con trampa de luz negra en los terrenos cultivados con agave tequilero, las especies del género Phyllophaga presentaron su mayor abundancia en el mes de junio, lo cual coincide con lo reportado por Magaña \& Rivera (1998) en el cerro la Campana en el municipio de Atenguillo y por López \& Rivera (1998) en Manantlán, Jalisco, donde este género fue abundante durante junio, pero su registro disminuyó considerablemente en julio. Phyllophaga ravida, Ph. dentex, Cyclocephala comata, C. lunulata, Strategus aloeus y Golofa pusilla estuvieron mejor representadas en junio y disminuyeron en julio y agosto.

$\mathrm{Al}$ reunir todos los registros de las especies recolectadas en las trampas de luz en las tres localidades de Jalisco aquí estudiadas es evidente que la mayor diversidad de adultos se concentra en el mes de junio, en relación muy directa con el inicio del periodo de lluvias, cuando estos coleópteros alcanzan su mayor representatividad anual, lo cual coincide con lo reportado por Rodríguez del Bosque (1993) que menciona que en el norte de Tamaulipas la emergencia y actividad de vuelo de los Melolonthinae y Anomalini están más relacionadas con patrones pluviales que con las fluctuaciones de temperatura.

Es evidente que los melolóntidos con larvas edafícolas son abundantes en el estado de Jalisco, debido a que es relativamente fácil obtener muestras grandes y representativas, ya que con una trampa de luz negra se obtuvo un promedio de 5,726 ejemplares por noche de trampeo, muy superior al reportado por Morón et al. (1998) quienes recolectaron un promedio de 1,660 individuos por noche en una región cañera del estado de Nayarit. Es oportuno resaltar que de las 20 especies registradas en éste trabajo, solo las larvas de Pelidnota virescens no se desarrollan en el suelo.

La abundancia y la riqueza específica fueron bastante diferentes en las tres localidades. La localidad donde se obtuvieron mas ejemplares $(46,882)$ y el mayor número de especies (17) de Melolonthidae fue Tepatitlán, pero el notable predominio de dos especies (Phyllophaga ravida y Paranomala cincta) que representan el $83.41 \%$ de la muestra local, sugiere un desequilibrio acentuado en la comunidad de escarabajos edafícolas. En San Juanito se capturaron 7,999 ejemplares que representan a 13 especies, entre las que predominan Phyllophaga ravida y $P h$. misteca, que son equivalentes al $72.13 \%$ de la muestra local, y podría estimarse que la comunidad edafícola está menos desequilibrada. En Ixtlahuacan se colectaron 2,381 escarabajos de 11 especies, entre los que predomina Diplotaxis trapezifera, que corresponde al 67.32 $\%$ de la muestra local, por lo que es factible que la comunidad de especies del suelo esté mas equilibrada. 
Sumando los resultados en las tres localidades estudiadas las especies más abundantes fueron Phyllophaga ravida (Blanchard) (58.7\%), Paranomala cincta (Say) (14.1\%), Phyllophaga misteca (Bates) (11.6), P. dentex (Bates) (2.5\%) y Paranomala hoepfneri (Bates) (1.8\%). De las cuales es posible que las larvas de Ph. ravida, $P h$. misteca y $P h$. dentex puedan ocasionar daños a las plantas pequeñas de agave tequilero, ya que se han calificado como rizófagas en el cultivo de maíz (Díaz et al. 2006, Castro-Ramírez et al. 2005). Diversos autores señalan que estas especies de Phyllophaga tienen ciclo de vida anual (Morón 1986, Morón et al. 1998, Nájera 1998, Aragón et al. 2005) y que en los estados de Chiapas y Puebla sus vuelos inician con las primeras lluvias.(Ramírez-Salinas \& Castro-Ramírez, 2000, Castro-Ramírez et al. 2005, Nochebuena \& Aragón 2005). Pero no se ha confirmado una asociación entre las larvas de tales especies y las raíces o las partes inferiores de los agaves, aunque Angelina (2004) localizó concentraciones considerables de larvas durante sus muestreos de suelo en parcelas con agaves en Tepatitlán donde coexistían numerosas herbáceas. Es probable que las larvas prefieran las raíces de las malezas y que sólo cuando disminuya mucho la humedad del suelo o desaparezcan las herbáceas, las larvas de algunas de estas especies busquen las raíces del agave.

Otra especie ligeramente abundante en Tepatitlán y San Juanito de Escobedo fue Strategus aloeus, "escarabajo rinceronte" cuyos adultos se han citado con frecuencia como causantes de daños en la parte basal de los agaves maduros (Pérez-Domínguez 2006), sin embargo en esta oportunidad no se confirmó la presencia de galerías en los agaves.

Debido al ritmo de expansión del cultivo del agave tequilero en Jalisco y el consecuente reemplazo de otros cultivos tradicionales, así como la remoción de las comunidades vegetales nativas, consideramos prudente recomendar que se continúen este tipo de estudios para monitorear las poblaciones de especies de Melolonthidae con adultos fitófagos o larvas rizófagas, y sobre todo iniciar investigaciones sobre la composición y dinámica del complejo gallina ciega en las parcelas sembradas con agave tequilero, para determinar cuales especies rizófagas pueden causar realmente daños en dicho cultivo, y cuales podrían catalogarse como benéficas por su capacidad para remover y enriquecer el suelo (Romero-López et al. 2010). También sería importante profundizar en el conocimiento de los hábitos y la dinámica poblacional de $S$. aloeus para disponer con oportunidad de los recursos necesarios para diseñar un programa de control en el probable caso de que se constituya en una plaga severa del agave.

AGRADECIMIENTOS. Se agradece a la empresa Tequila Sauza S.A. de C.V. y Casa Herradura por el apoyo económico y logístico a los proyectos: "Biología, efectividad biológica de insecticidas y ecología química del picudo del agave" y "Manejo Integrado de Plagas del Agave", al TAMU-CONACyT Collaborative Research Grant Program por el apoyo al proyecto: "Developing environmentally friend management technologies for emerging insects pest of tequila agave," y al Ing. Saúl Montecinos por el apoyo técnico y logístico en los muestreos realizados. 
Lugo-Garcia et al.: Coleoptera Melolonthidae de la zona agavera de Jalisco

\section{LITERATURA CITADA}

Angelina, B. R. 2004. Complejo gallina ciega (Coleoptera: Melolonthidae) en Agave tequilana Weber Var. Azul en Tepatitlán, Jalisco, México. Tesis de Maestría, Instituto de Fitosanidad, Colegio de Postgraduados, Montecillo, Estado de México. 70 pp.

Aragón, G. A. \& M. A. Morón. 1998. Evaluación del daño ocasionado por el complejo "gallina ciega" (Coleoptera: Melolonthidae) en el estado de Puebla, pp. 143-149. In: M. A. Morón y A. Aragón (Eds.). Avances en el estudio de la diversidad, importancia y manejo de los coleópteros edafícolas americanos. Publicación especial de la Benemérita Universidad Autónoma de Puebla y la Sociedad Mexicana de Entomología, A. C. Puebla, México.

Aragón G. A. \& M. A. Morón. 2000. Los coleópteros Melolonthidae asociados a la rizosfera de la caña de azúcar en Chietla, Puebla, México. Folia Entomológica Mexicana, 108: 79-94.

Aragón, G. A., M. A. Morón, A. M. Tapia R. \& G. R. Rojas. 1998. Las especies de Coleoptera Melolonthidae relacionadas con plantas cultivadas en el estado de Puebla, México, pp. 131-142. In: M. A. Morón y A. Aragón G. (Eds.). Avances en el estudio de la diversidad, importancia y manejo de los coleópteros edafícolas americanos. Publicación especial de la Benemérita Universidad Autónoma de Puebla y la Sociedad Mexicana de Entomología, A. C. Puebla, México.

Aragón, G. A., M. A. Morón, A. M. Tapia R. \& R. Rojas. 2001. Fauna de Coleoptera Melolonthidae en el Rancho "La Joya", Atlixco, Puebla, México. Acta Zoológica Mexicana (n.s.), 83: 143-164.

Aragón, G. A., M. A. Morón, J. F. López O. \& L. M. Cervantes. 2005. Ciclo de vida y conducta de adultos de cinco especies de Phyllophaga Harris, 1827 (Coleoptera: Melolonthidae: Melolonthinae). Acta Zoológica Mexicana (n.s), 21: 87- 99.

Aragón, G. A., C. D. Nochebuena T., M. A. Morón \& J. F López-Olguín. 2008. Uso de trampas de luz fluorescente para el manejo de la gallina ciega (Coleoptera: Melolonthidae) en maíz (Zea mays L.). Agrociencia, 42: 217-223.

Aragón, G. A., B. C. Pérez-Torres, M. A. Morón, J. F. López \& A. M. Tapia. 2006. Desarrollo biológico y comportamiento de cinco especies del género Phyllophaga (Harris, 1827) (Coleoptera: Melolonthidae; Melolonthinae), pp. 49-62. In: A. E. Castro R. M. A. Morón y A. Aragón G. (Eds.). Diversidad, Importancia y Manejo de Escarabajos Edaficolas. Publicación especial de El Colegio de la Frontera Sur, la Fundación PRODUCE Chiapas, A. C. y la Benemérita Universidad Autónoma de Puebla, México.

Blackwelder, R. E. \& R. H. Arnett, Jr. 1974. Checklist of Scarabaeidae of Canada, United States, Mexico, Central America and the West Indies. Vol. 1 Pt. 3 (red version) BRIA Latham, N. Y. 120 pp.

Castro-Ramírez, A. E., G. H. Delfín, V. Parra \& M. A. Morón. 2005. Fauna de Melolóntidos (Coleoptera: Scarabaeoidea) asociados al maíz (Zea mays 1.) en Los Altos de Chiapas, México. Folia Entomológica Mexicana, 44: 339-365.

Deloya, C. \& M. A. Morón. 1994. Coleópteros Lamelicornios del distrito de Jojutla, Morelos, México. Listados Faunísticos de México, $V$. Instituto de Biología, UNAM, pp. 1-49.

Díaz, M. P., M. B. Nájera, R. Ledesma, O. Rebolledo, H. F. Flores \& J. A. Martínez. 2006. Especies de gallina ciega (Coleoptera: Melolonthidae) y su asociación con factores agroclimáticos y de manejo del maíz en los altos de Jalisco, México. Fitosanidad, 10: 209-215.

Endrödi, S. 1985. The Dynastinae of the Word. Dr. W. Junk Publ. Dordrecht. 800 pp., XLVI plates.

López, V. M. \& L. E. Rivera. 1998. Abundancia estacional de los coleópteros Melolonthidae) asociados a un bosque mesófilo de montaña en la estación científica las Joyas, sierra de Manantlán, Jalisco, México, pp. 61-70. In: M. A Morón y A. Aragón (Eds.). Avances en el estado de la diversidad, importancia y manejo de los coleópteros edafícolas americanos. Publicación especial de la Benemérita Universidad Autónoma de Puebla y la Sociedad Mexicana de Entomología, A. C., Puebla, México. 
Luna, Z. R. 1991. La Historia del Tequila, de sus regiones y sus hombres. Editorial. Consejo Nacional para la Cultura y las Artes. México D. F. 302 pp.

Magaña, C. B. \& L. E. Rivera. 1998. Abundancia estacional de los coleópteros nocturnos de la familia Melolonthidae (Insecta: Lamellicornia), asociados a un bosque de pino-encino en el Municipio de Atenguillo, Jalisco, México, pp. 51-60. In: M. A Morón y A. Aragón (Eds.). Avances en el estado de la diversidad, importancia y manejo de los coleópteros edaficolas americanos. Publicación especial de la Benemérita Universidad Autónoma de Puebla y la Sociedad Mexicana de Entomología, A. C., Puebla, México.

Méndez A. M. J., A. E. Castro, R. Alvarado, C. Pacheco \& C. Ramírez. 2005. Eficacia de dos tipos de recolecta para registrar la diversidad de Melolóntidos Nocturnos (Coleoptera: Scarabaeoidea). Acta Zoológica Mexicana (n. s.), 21: 109-124.

Morón, M. A. 1986. El género Phyllophaga en México. Morfología, distribución y sistemática supraespecífica (Insecta: Coleoptera). Publicación 20. Instituto de Ecología, México. 344 pp.

Morón, M. A. 1994. Fauna de Coleoptera Lamellicornia en las montañas del noreste de Hidalgo, México. Acta Zoológica Mexicana (n.s.), 63: 7-59.

Morón, M. A. 2003. Diversidad, distribución e importancia de las especies de Phyllophaga Harris en México (Coleoptera: Melolonthidae), pp. 1-27. In: A. Aragón, M. A. Morón y A. Marín J. (Eds.). Estudios sobre coleópteros del suelo en América. Publicación especial de la Benemérita Universidad Autónoma de Puebla.

Morón, M. A. 2004. Escarabajos 200 millones de años de evolución. Instituto de Ecología, A. C.. y Sociedad Entomológica Aragonesa, Zaragoza, España. 204 pp.

Morón, M. A., C. Deloya, S. Hernández \& A. Ramírez. 1998. Fauna de Coleoptera Lamellicornia de la región de Tepic, Nayarit, México. Acta Zoológica Mexicana (n. s.), 75: 73-116.

Morón, M. A., S. Hernández \& A. Ramírez. 1996. El complejo “gallina ciega” (Coleoptera: Melolonthidae) asociado con la caña de azúcar en Nayarit, México. Folia Entomológica Mexicana, 98: 1-44.

Morón, M. A., B. C. Ratcliffe \& C. Deloya. 1997. Atlas de los Escarabajos de México (Coleoptera: Lamellicornia) volumen 1, familia Melolonthidae. CONABIO-Sociedad Mexicana de Entomología, A. C. México. 280 pp.

Morón, M. A. \& L. E. Rivera. 2005. Phyllophaga (Phytalus) aliciae, una nueva especie de Jalisco, México. (Coleoptera: Melolonthidae: Melolonthinae). Folia Entomológica Mexicana, 44 (supl. 1): 9-13.

Nájera, M. B. 1993. Coleópteros rizófagos asociados al maíz de temporal en el Centro del Estado de Jalisco, México, pp. 143-154. In: M. A. Morón (Ed.). Diversidad y manejo de plagas subterráneas. Publicación especial de la Sociedad Mexicana de Entomología e Instituto de Ecología, Xalapa, México.

Nájera, R. M. B. 1998. Diversidad y abundancia del complejo "gallina ciega" (Coleoptera: Melolonthidae) en agroecosistemas de maíz en la región templada de Michoacán, México, pp. 96-106. In: M. A Morón y A. Aragón (Eds.). Avances en el estado de la diversidad, importancia y manejo de los coleópteros edaficolas americanos. Publicación especial de la Benemérita Universidad Autónoma de Puebla y la Sociedad Mexicana de Entomología, A. C., Puebla, México.

Navarrete-Heredia J. L., L. Delgado \& H. E. Fierros-López. 2001. Coleoptera Scarabaeoidea de Jalisco, México. Dugesiana, 8: 37-93.

Nochebuena, T. C. D. \& A. Aragón. 2005. Control del complejo "gallina ciega" (Coleoptera: Melolonthidae) utilizando trampas de luz flúor, en la zona maicera de Santa Cruz Alpuyeca, Cuautinchán, Puebla, pp. 590-594. In: A. Morales M., A. Mendoza E., M. P. Ibarra G.y S. Stanford C. (Eds.). Entomología Mexicana. Vol. 4. Sociedad Mexicana de Entomología y Colegio de Postgraduados. México. 
Pacheco F. C., A. E. Castro, M. A. Morón \& B. Gómez. 2008. Fauna de escarabajos Melolóntidos (Coleoptera: Scarabaeoidea) en el municipio de Villaflores, Chiapas, México. Acta Zoológica Mexicana (n.s.), 24: 139-168.

Pérez-Domínguez, J. F. 2006. Importancia del escarabajo rinoceronte Strategus aloeus (L.) (Coleoptera: Scarabaeidae) como plaga del cultivo de agave en Jalisco, México, pp. 181-194. In: A. E. Castro R. M. A. Morón y A. Aragón G. (Eds.). Diversidad, Importancia y Manejo de Escarabajos Edaficolas. Publicación especial de El Colegio de la Frontera Sur, la Fundación PRODUCE Chiapas, A. C. y la Benemérita Universidad Autónoma de Puebla, México.

Ramírez-Salinas, C. \& A. E Castro-Ramírez. 2000. El complejo "gallina ciega" (Coleoptera: Melolonthidae) en el cultivo de maíz en El Madronal, municipio de Amatenango del Valle de Chiapas, México. Acta Zoológica Mexicana (n.s.), 79: 17-41.

Ramírez, S. C. \& A. E. Castro. 2006. Ciclo de vida de dos especies rizófagas de "gallina ciega" (Coleoptera Melolonthidae) en los Altos de Chiapas, pp. 37-48. In: A. E. Castro R. M. A. Morón y A. Aragón G. (Eds.). Diversidad, Importancia y Manejo de Escarabajos Edaficolas. Publicación especial de El Colegio de la Frontera Sur, la Fundación PRODUCE Chiapas, A. C. y la Benemérita Universidad Autónoma de Puebla, México.

Ramírez, S, C., M. A. Morón \& A. E. Castro. 2004. Descripción de los estados inmaduros de tres especies de Paranomala, Ancognatha y Ligyrus (Coleoptera: Melolonthidae: Rutelinae y Dynastinae) con observaciones de su biología. Acta Zoológica Mexicana (n.s.), 20: 67-82.

Rodríguez del Bosque, L. A. 1993. Abundancia estacional y ecología de coleópteros rizófagos: un estudio durante 15 años en agroecosistemas del norte de Tamaulipas, pp. 7-15. In: Morón (Comp.). Diversidad y Manejo de plagas Subterráneas. Publicación Especial de la Sociedad Mexicana de Entomología e Instituto de Ecología de Xalapa, Veracruz, México.

Romero-López, A. A., M. A. Morón, A. Aragón \& F. J. Villalobos. 2010. La "gallina ciega" (Coleoptera: Scarabaeoidea: Melolonthidae) vista como un "ingeniero del suelo". Southwestern Entomologist, 35: 331-343.

\section{APÉNDICE 1}

Clave basada en machos para separar las especies de melolóntidos nocturnos recolectados con trampa de luz negra en el cultivo de agave en el estado de Jalisco, México.

1 Labro amplio, bien desarrollado, con el borde anterior más o menos engrosado y visible bajo el clípeo. Las dos uñas de cada meso y metatarso sencillas, dentadas o bífidas, de igual o diferente longitud y grosor. . . . . . . . . . . . . . . . . . 2

1' Labro reducido, laminar o membranoso con el borde anterior muy delgado y oculto bajo el clípeo. Las dos uñas de cada meso y metatarsos sencillas de igual longitud y grosor. . .

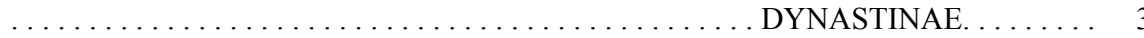

2 Borde exterior de las mandíbulas usualmente expuesto a los lados del clípeo. Las dos uñas de cada par de tarsos con diferente longitud, forma y grosor. . . RUTELINAE. . . 9

$2^{\prime} \quad$ Borde exterior de las mandíbulas usualmente oculto bajo el clípeo. Las dos uñas de cada pro y metatarsos con la misma longitud, forma y grosor. . . . MELOLONTHINAE ... 13

3 Cabeza con tubérculos, quilla o prominencias notables. . . . . . . . . . . . 4

3' Cabeza sin prominencias notables. . . . . . . . . . Cyclocephalini. ..... 5 
4 Élitros amarillos, anaranjados, pardo amarillentos o pardo obscuros. Machos con un tubérculo pronotal redondeado, dirigido hacia arriba, provisto con algunas sedas amarillas en su lado anterior. Longitud corporal 24-29 mm. . . . . . . . . Golofa pusilla Arrow

4' Élitros rojizos o negro brillantes. Meso y metatibias usualmente con los ápices ensancha-

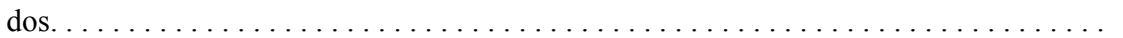

5 Pronoto y élitros sin manchas obscuras. Machos con la maza antenal dos veces más larga que todos los artejos precedentes. Longitud corporal $10-11 \mathrm{~mm} . \ldots \ldots \ldots \ldots \ldots \ldots$ $\ldots \ldots \ldots \ldots \ldots \ldots \ldots \ldots \ldots \ldots \ldots \ldots \ldots \ldots \ldots \ldots \ldots \ldots$ Cyclophala sinaloae $\mathrm{H}$ y E.

5 Pronoto con manchas obscuras. Maza antenal mas corta que el flagelo. . . . . . . . . .

6 Pronoto y élitros con manchas obscuras irregulares. Meso y metatarsos con las dos uñas sencillas y de tamaño similar. Dorso con abundantes sedas finas. Longitud corporal 23-

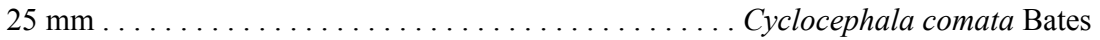

6' Pronoto con dos franjas longitudinales obscuras y sinuosas, irregulares. Élitros con dos series transversales de 4 manchas obscuras y una franja obscura a los lados de la sutura. Dorso sin sedas. Longitud corporal 22-25 mm. . . . Cyclocephala lunulata Burmeister

7 Ápice de las metatibias recto o ligeramente festonado, con dentículos. Clípeo con el ápice truncado sin dentículos. Pronoto completamente convexo. Borde externo de las protibias con tres dentículos grandes y dos dentículos pequeños intercalados entre los grandes. Coloración pardo obscura brillante. Longitud corporal 19-21 mm.

Ápice de las metatibias con dentículos gruesos y tan largos como la mitad del segundo artejo tarsal. Color pardo obscuro o rojizo. Dimorfismo sexual normalmente acentuado. .

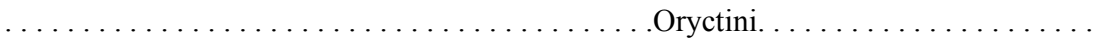

Pronoto con una depresión central muy amplia y profunda, en los machos está flanqueada por tres proyecciones o tubérculos grandes o anchos, más o menos dirigidos hacia el frente. Cabeza con dos tubérculos transversales. Longitud corporal 30-54 mm. ........ $\ldots \ldots \ldots \ldots \ldots \ldots \ldots \ldots \ldots \ldots \ldots \ldots \ldots \ldots \ldots \ldots \ldots \ldots \ldots \ldots \ldots$ Strategus aloeus (Linné)

8' Pronoto del macho con un lóbulo pequeño proyectado anteriormente desde el margen superior de una cavidad somera y amplia. Cabeza con un cuerno corto. Longitud corporal

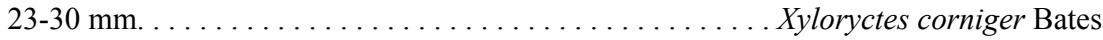

9 Margen exterior de los élitros con un borde membranoso estrecho pero conspicuo. Antenas formadas por 9 artejos. . . . . . . . . . . . . .

9' Margen exterior de los élitros sin borde membranoso. Antenas formadas por 10 artejos (Rutelini). Clípeo semitrapezoidal con puntuación fina y regular. Espolones metatibiales estrechos y agudos. Región dorsal iridescente, amarillenta, blanquecina, nacarada muy brillante. Regiones ventrales verde metálico. Longitud corporal 18-25 mm. ..........

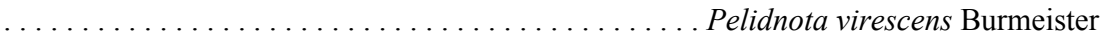

10 Élitros con hileras de puntos profundos, claramente marcadas. Maza antenal ensanchada y más larga que el flagelo. Coloración dorsal parda rojiza con intensos reflejos verde metálico. Longitud corporal 10-13 mm. .......... Paranomala micans (Burmeister)

10' Élitros con hileras de puntos pequeños, someros, irregularmente marcadas. Maza antenal estrecha y tan larga como el flagelo. Longitud corporal mayor a $15 \mathrm{~mm} . . . \ldots \ldots \ldots$. . 
11 Pronoto bicolor, amarillento o blanquecino, con manchas o franjas pardas. Élitros amarillentos con manchas o franjas oscuras contrastantes . . . . . . . . . . . . . 12

11' Pronoto unicolor, pardo obscuro o pardo rojizo con intensos reflejos verde metálico. Élitros amarillentos sin manchas contrastantes. Placa pigidial y esternitos obscuros. . . . . . .

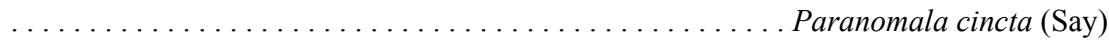

12 Pronoto pardo rojizo con los márgenes laterales amarillos. Élitros amarillentos con manchas casi de color negro. . . . . . . . . . . . Paranomala inconstans (Burmeister)

12' Pronoto amarillo pajizo o blanquecino, con una mancha parda obscura o rojiza irregular en la parte media anterior. Élitros pajizos o blanquecinos con los márgenes y las regiones humerales pardo obscuras. ..................... Paranomala hoepfneri (Bates)

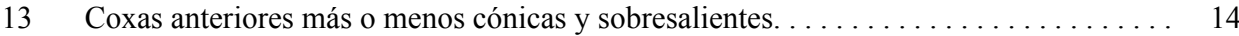

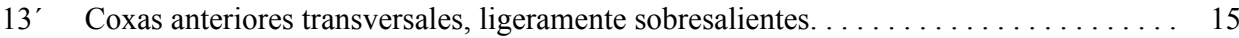

14 Placa pigidial pequeña semitriangular rugosa. Pronoto punteado y glabro. Cuerpo glabro. Coloración parda obscura rojiza. Longitud corporal 7.5-9.5 mm. ............ Diplotaxis trapezifera Bates

14' Placa pigidial grande, semitriangular. Pronoto más ancho que largo. Tegumento dorsal y ventral pardo rojizo o pardo obscuro, cubierto con sedas escamiformes amarillentas. Los élitros muestran un patrón simétrico de manchas irregulares. Longitud corporal 9-10 mm. . . . . . . . . . . . . . . . . . . . . . . Isonychus arizonensis Howden

15 Mesotibias con una proyección dentiforme en la parte media de su lado externo. Propigidio con un surco medio longitudinal bien marcado. Sin dimorfismo sexual aparente. . . .

15' Mesotibias con una carina completa transversal u oblicua en la parte media de su lado externo. Propigidio sin surco longitudinal medio. Dimorfismo sexual más o menos acen-

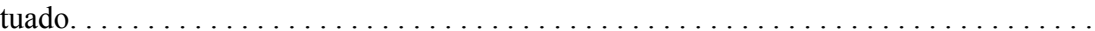

16 Maza antenal masculina casi tan larga como el escapo. Élitros ensanchados en sus dos tercios posteriores. Longitud corporal $26.5-32.0 \mathrm{~mm} \ldots \ldots \ldots \ldots \ldots \ldots \ldots \ldots \ldots$

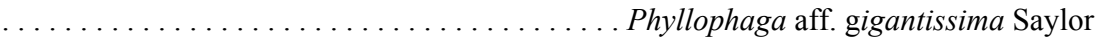

16' Maza antenal masculina más larga que el escapo. La mitad posterior de los élitros casi tan ancha como la mitad anterior. Longitud corporal $21-30 \mathrm{~mm} . \ldots \ldots \ldots \ldots \ldots$. ..................... Phyllophaga aff nogueirai Warner \& Morón

17 Uñas tarsales con el diente intermedio ampliamente separado del ápice. . . . . . . . .

$17^{\prime}$ Unas tarsales con el diente intermedio flanqueado por escotaduras estrechas y profun-

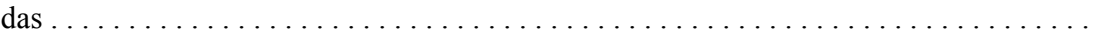

18 Coloración dorsal parda rojiza. Base del pronoto y de los élitros con abundantes sedas erectas y cortas. Proyecciones apicales de los parámeros alargados y aguzados. Longitud

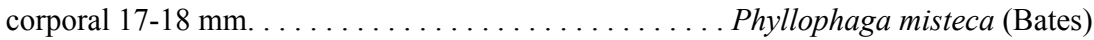

18' Coloración dorsal parda obscura. Base del pronoto y de los élitros con numerosas sedas erectas, muy largas y gruesas. Proyecciones apicales de los parámeros cortas y redondea-

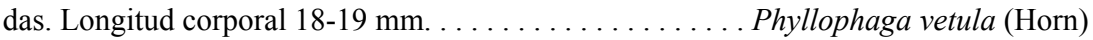


19 Maza antenal formada por cinco lamelas. Pronoto, élitros y placa pigidial brillante, glabros o con pocas sedas aisladas. Pigidio sin sedas, con puntos pequeños y prominente hacia el ápice. . . . . . . . . . . . . . . Phyllophaga polyphylla (Bates)

19' Maza antenal formada por tres lamelas. Pronoto, élitros y placa pigidial ligeramente brillantes. Pigidio con sedas cortas.

20 Pronoto y élitros con abundantes sedas cortas más o menos separadas. El pigidio convexio, con sedas cortas en la base y largas en el ápice. Ápice de los parámeros inclinado hacia la base. . . . . . . . . . . . . . . . . . Phyllophaga

$20^{\prime}$ Pronoto y élitros prácticamente glabros o con algunas sedas aisladas. Pigidio poco convexo, con algunas sedas cortas. Ápice de los parámeros recto. 